\title{
Designing Usable Interfaces with Cultural Dimensions
}

\author{
Gabrielle Ford ${ }^{1}$ and Paula Kotzé ${ }^{2}$ \\ ${ }^{1}$ School of Information Systems and Technology, University of Kwa-Zulu Natal, \\ King George V Avenue, Durban, South Africa \\ fordg1@ukzn.ac. za \\ http://www.ukzn.ac.za \\ ${ }^{2}$ School of Computing, University of South Africa, P O Box 392, Unisa, 0003, South Africa \\ kotzep@unisa.ac.za \\ http://www.cs.unisa.ac.za
}

\begin{abstract}
There are as many arguments against as supporting the accommodation of culture into user interface design. One argument suggests that it is necessary to match the subjective cultural profile of the interface to the cultural profile of the users in order to enhance usability and performance. In contrast, we argue that the interface design characteristics required to design interfaces to accommodate one side of four of the five cultural dimensions proposed by Hofstede will result in an increase in usability for all users, irrespective of the users' cultural profile. Secondary data analysis of a prior experiment somewhat supported our argument, but we conclude that further research into the effects of Hofstede's cultural dimensions is required before our hypotheses can be accepted.
\end{abstract}

\section{Introduction}

The influence of culture on usability is a controversial issue in the field of humancomputer interaction. Those that are in support of accommodating culture into the design of user interfaces do not seem to agree on whether to incorporate objective culture, subjective culture or both into user interface design. Furthermore, the use of cultural dimension models as a way of managing the subjective aspects of user interface design has been severely criticized.

In contrast to the arguments against the use of cultural dimension models, and in particular, Hofstede's [1] model, we argue that the interface design characteristics required to design interfaces to accommodate high power distance, high uncertainty avoidance, masculinity and short-term orientation, would provide a generally more usable interface than one that is designed to accommodate the opposing sides of these dimensions.

This paper presents initial evidence in support of our argument that interfaces that display characteristics relevant to specific sides of four of Hofstede's cultural dimensions increase usability for all users. Section 2 briefly reviews the concept of culture and approaches to culturalisation, while Section 3 focuses on culture in the context of usability and interface design. Section 4 presents details on our research and an experiment conducted. Section 5 concludes and mentions future research. 


\section{Culture}

To better understand the concept of culture, and how it is related to human-computer interaction, we review below the definitions, metamodels and models that have been proposed in the literature.

\subsection{Definitions}

There are many definitions of culture in the literature, but there is no agreement on a specific definition of culture [2,3]. Some examples of such definitions include:

- Culture is conceptualized as a 'system of meaning that underlies routine and behaviour in everyday working life' [4, p 122].

- Culture 'includes race and ethnicity as well as other variables and is manifested in customary behaviours, assumptions and values, patterns of thinking and communication style' [5, p 49].

- 'Culture is communication, and communication is culture' [6, p 186].

- Culture is 'the collective programming of the mind that distinguishes the members of one group or category of people from another, where the mind stands for thinking, feeling and acting, with consequences for beliefs, attitudes and skills' $[1, \mathrm{p} 5]$.

Most of the above definitions refer to culture as influencing the way in which communication takes place. Using the computer to perform tasks requires communication between the user and the system, particularly when using an interactive system. Consequently, for the purposes of this paper, we define culture as the patterns of thinking, feeling and acting that influence the way in which people communicate amongst themselves and with computers.

\subsection{Metamodels of Culture}

Metamodels of culture provide a high-level view of the overriding philosophies surrounding the concept of culture, by defining different layers of culture [2]. Four metamodels have been proposed in the literature, including the Onion Model, the Pyramid Model, the Iceberg Model and the Objective and Subjective Culture Model [2]. For the purposes of this research, we will adopt the latter metamodel.

The Objective Culture and Subjective Culture Model, developed by Stuart and Bennett [2], identifies only two layers of culture, objective culture and subjective culture. Objective culture is the institutions and artefacts of a culture, such as its economic system, social customs, political structures and processes, arts, crafts and literature' [2, p 43]. Objective culture is visible, easy to examine and tangible, as it is represented in terms of text orientation, date and number formats, colour and language [7]. In contrast, subjective culture is 'the psychological features of a culture, including assumptions, values, and patterns of thinking' [2, p 43]. Subjective culture is difficult to examine because it operates outside of conscious awareness, for example, in the way in which people accept or reject uncertainty [1], similarities and differences in power and authority [1,8], and the amount of emotions that people express when dealing with others [9]. 
Objective culture is abstract, because it is an externalization of subjective culture. Subjective culture is what is real and concrete. However, objective culture tends to be treated as more real and concrete than its source, subjective culture [2].

\subsection{Models of Culture}

The metamodels of culture form the basis for the development of different models of culture. These models provide a more detailed view of culture, by identifying a number of cultural dimensions that are used to organise cultural data [10]. Hoft [2] describes four models of culture, developed by Victor [8], Hall [6], Trompenaars [9] and Hofstede [1], which are summarised in Table 1.

Table 1. Cultural models and their dimensions

\begin{tabular}{ll}
\hline Victor [8] & Hall [6] \\
\hline - Language & - Speed of Messages \\
- Environment and Technology & - Context \\
- Social Organisation & - Space \\
- Contexting & - Time \\
- Authority Conception & - Information Flow \\
- Nonverbal Behaviour & - Action Chains \\
- Temporal Conception & \\
\hline Trompenaars [9] & Hofstede [1] \\
\hline - Universalism vs. Particularism & - Power Distance \\
- Neutral or emotional & - Masculinity vs. Femininity \\
- Individualism vs. Collectivism & - Individualism vs. Collectivism \\
- Specific vs. Diffuse & - Uchievement vs. Ascription \\
- Time & - Time Orientation \\
- Environment & \\
\hline
\end{tabular}

Hofstede [1] focuses his model on determining the patterns of thinking, feeling and acting that form a culture's mental programming. He conceptualized culture as 'programming of the mind' in the sense that certain reactions were more likely in certain cultures than in others, based on differences between the basic values of the members of different cultures [11].

As reflected in Table 1, Hofstede identified five cultural dimensions that can be used to distinguish among different cultures [12]. All five of Hofstede's dimensions relate to subjective culture, and many of these dimensions also appear in the other three models summarised in Table 1. Each of these dimensions is a dichotomy, in that there are two opposing sides to each dimension. The design characteristics of interfaces that are designed to accommodate each side of these dimensions is presented in Section 3.1.3. The character traits expected of users displaying each side of each dimension is presented below $[2,10,12,13]$ : 
- Power distance refers to the extent to which less powerful members of a society or group of people expect and accept unequal power distribution within that group. High power distant people are afraid to express disagreement with people in authority such as bosses, parents and teachers. Low power distant people have little difficulty in approaching and contradicting their superiors.

- Uncertainty avoidance is the way in which people cope with uncertainty and risk. High uncertainty avoidant users tend to be emotional and aggressive, avoid ambiguous situations, prefer to work in a structured and predictable environment, and consider differences to be threatening and dangerous. It is also believed that high uncertainty avoidant users would prefer to work within a team environment, as this would serve as a support structure in times of uncertainty. In contrast, low uncertainty avoidant users can accept that superiors do not have all the answers, that there may be more than one correct answer, and are curious about differences.

- Masculinity vs. femininity refers to gender roles, not physical characteristics, and is primarily characterized by the levels of assertiveness or tenderness in the user. Masculine users tend to be assertive, competitive and tough. Their work goals include high earnings, recognition, advancement and challenge. Feminine users focus on home, children and people. Their work goals include good relations with supervisors, peers and subordinates, good living and working conditions with sense of security.

- Individualism vs. collectivism relates to the role of the individual and the group, and is characterized by the level of ties between an individual in a society. Individualist users are expected to look after themselves and their immediate family, but no one else. They value personal time, freedom and challenge, material rewards, honesty and truth, talking things out, maintaining self-respect, and the right to privacy and personal opinion. In contrast, collectivist users are integrated into strong, cohesive groups that protect them in exchange for unquestioning loyalty. Collectivists value training and skills, and group achievement rather than personal recognition. Harmony is valued more than truth and honesty. They are comfortable with an invasion of privacy and restrictions on personal opinions.

- Time orientation relates to people's concern with the past, present and future. In essence, short-term oriented people are concerned with the past and the present, while long-term oriented people are concerned more with the future. Long-term oriented users believe that a stable society requires unequal relations, and that older people and men have more authority than younger people and women. They value trying to acquire skills and education, working hard and being frugal. They are prepared to persevere and display a lot of patience in understanding new things. In contrast, short-term oriented users believe in an equality of relationships, and emphasize individualism. They value reciprocity of favours, gifts and greetings, and the ability to achieve quick results.

\section{Effects of Cultural Dimensions on Usability and Interface Design}

As culture influences the way in which people interact in general, culture will also influence the way in which people will interact with computers. Using interactive 
systems to perform tasks requires communication between the system and the user. People learn patterns of thinking, acting and communicating from living in a specific social environment, normally typified by national culture [14]. As such, culture partially predetermines a person's communication preferences and behaviours. Communication style, which reflects how a person sends and interprets messages, represents the overall patterns and values of a culture. As the user interface is the means by which the user and the computer interact [15], it stands to reason that the interface should facilitate users to use their particular communication styles [14]. Consequently, global interfaces need to accommodate a diversity of communication styles to provide support for the cultural diversity of the users.

Diversity in culture is particularly relevant where global interfaces take the form of websites [7, 16, 17, 18, 19, 20, 21]. The advent of the World Wide Web (WWW) has resulted in a fundamental technical context of use that now needs to be taken into consideration, namely the difference between traditional software applications and web applications.

From a cross-cultural usability perspective, the primary difference between traditional software and web-based interfaces is that websites are constantly addressing different cultural audiences simultaneously [18]. Within the global information technology environment, cross-cultural usability of websites is about making websites an effective means of communication between a global web site owner and a local user [11]. Because users differ across regional, linguistic and country boundaries, their expectations of websites are driven primarily by their local cultural perspectives. Consequently, user reactions become more predictable and understandable when the user's cultural perspective is taken into account $[14,17]$. Websites need to display 'culturability', that is, designing the interface to accommodate the cultural preferences and biases to increase the usability of the interface and the product [17].

\subsection{Approaches to Culturalisation}

Culturalisation, or preparing a product for use by diverse cultures, requires two steps: internationalization and localization. Internationalisation involves identifying the culturally specific elements of the product, and localization involves substituting those culturally specific elements with a local content [22].

Traditionally, the approaches to culturalisation seemed to focus primarily on objective cultural issues rather than subjective culture.

The Objective Culture Approach. The objective cultural approach concludes that, when dealing with human-computer interaction, meaning is the central issue in culture [23]. Supporters of this approach suggest that designers need only cater for cultural diversity by ensuring that the intended meaning of user interface representations, such as symbols, icons and language, are translated to suit the target cultures, so that they are understood correctly. Thus, this approach is based on the premise that it is the objective, rather than the subjective, cultural aspects that are important. The culturalisation process has concentrated primarily on the translation of the objective cultural aspects [24], such as language and date and time formats [22, 23], to avoid potentially harmful misunderstandings. 
The Subjective Culture Approach. It has been argued that whilst objective culture is important, it is also necessary for the interfaces to reflect the values, ethics and morals of the target users [22], in order to make the users more comfortable and accepting of the interfaces [25]. These aspects relate to subjective culture [24], and go beyond the 'surface manifestations of culture that have been widely accepted' [11, p 89]. Del Galdo and Nielsen [7] clearly support this by pointing out that there are three levels of internationalization, namely:

1. Displaying the native language, character set and notations.

2. Translating the user interface and documentation so that it is understandable and usable.

3. Matching the user's cultural characteristics, which goes beyond avoiding offensive icons and must accommodate the way business is conducted and the way people communicate.

Essentially, this approach is based on the premise that culture is about how individuals behave and respond, their beliefs and values, and therefore it is also necessary to reflect subjective culture in the design of interfaces [24]. Consequently, this approach suggests that the interface should be designed to match the users' cultural profile [20].

Accommodating Subjective Culture into the Design of Interfaces. Marcus [26] developed a set of guidelines for accommodating Hofstede's cultural dimensions into the design of user interfaces. These are described below.

- Power distance: Interfaces that display high power distance characteristics should provide highly structured access to information, prominence should be given to leaders, security measures should be both explicit and enforced, and there should be a strong focus on authority. The opposite holds true for low power distant sites.

- Uncertainty avoidance: Interfaces that display high uncertainty avoidance characteristics should focus on the prevention of user error by providing minimal menu options, simple and descriptive help facilities, and a navigation structure that is focused on preventing users from getting lost. Colours, sounds and images should be used to reinforce the messages. In contrast, low uncertainty avoidant interfaces should encourage user exploration; provide many menu options, and use colours, sounds and images to provide additional information.

- Masculinity vs. femininity: Interfaces that are oriented towards the masculine side of this dimension should be focused on allowing for quick results for limited tasks. The navigation structure should support user exploration and control. The content should be suggestive of a challenge for the user to master something, and cater for explicit distinctions between genders and age groups. Graphics and animations should be used for utilitarian purposes. In contrast, feminine oriented interfaces should use aesthetic appeal and poetry as a way of gaining users' attention. There is a blurring of gender roles. In particular, feminine oriented interfaces should support mutual cooperation and the exchange of ideas and support.

- Individualism vs. collectivism: Individualist interfaces should use images of materialism and consumerism to denote success, and youth, action and individuals to gain the users' attention. The content should be focused on personal achievement, 
new and unique products and concepts, and contain or encourage controversy and personal opinions. Users should not be required to provide personal information. In contrast, collectivist sites should use images of the achievement of socio-political agendas to denote success, and experienced, aged leaders and groups of people to gain the user's attention. The content should be focused on group achievement, history and tradition, and contain official slogans while discouraging personal opinions.

- Time orientation: Short-term oriented user interfaces should be structured in a way that allows users to complete tasks quickly. Rules should be used to verify the credibility of information, and information content should be based on truth and certainty of beliefs. In contrast, long-term oriented interface navigation style and content can be more complex, as users will persevere until they gain an understanding. Long-term oriented websites should contain content that is of practical value, and can use relationships to verify the credibility of the information.

However, the use of Hofstede's cultural dimensions model of managing the subjective aspects of cross-cultural interface design has been severely criticized as being too stereotypical [23] or rigid [16]. In addition, previous attempts to apply Hofstede's model to usability has resulted in conflicting and therefore inconclusive findings. For example, Gould et al. [27] found that Malaysian websites contain links on the home page to website administration, which correlates well with the high power distance reported [1] for Malaysia. However, this does not explain why low power distance countries such as the US also contain such links on their websites. In contrast, Forer and Ford [28] reported that accommodating for the user's cultural profile enhanced performance. Consequently, until better proof of their relevance to website design is provided, Fitzgerald [29] suggests that cultural dimension models should be used with care.

\section{Research on the Influence of Cultural Dimensions on Usability}

In contrast to the arguments put forward against the use of cultural models in general and against the use of Hofstede's cultural model [1] in particular, we believe that the inherent characteristics of a specific side of four of the five cultural dimensions proposed by Hofstede provide a generally more usable interface than the opposing side of the same dimension. For example:

- High uncertainty avoidant sites are designed to reduce uncertainty. According to Marcus [26], the design should provide clear and familiar metaphors, simple, clear articulation and limited menu options, simple and limited navigation controls, precise and detailed feedback of status, simple and clear imagery and highly redundant coding. All these characteristics would naturally cater for more accurate and speedier completion of tasks. This could also increase satisfaction levels as users would feel that the task had been accomplished quickly and correctly.

- Masculine site design incorporates similar characteristics to those of high uncertainty avoidant sites. For example, masculine sites should be designed to provide limited navigation choices, and high-level executive views, and are goal 
and work-oriented [26], thus providing for quick results of limited tasks. These characteristics would also naturally increase the speed and accuracy levels obtained, thereby possibly also increasing satisfaction levels.

- High power distant sites should also provide limited navigation choices, and wizards or guides to assist with navigation [26], thereby increasing the speed, accuracy and satisfaction levels obtained.

- Short-term time orientation design incorporates similar characteristics to those of masculine and high uncertainty avoidance site design. For example, short-term sites should be designed to provide bread-crumb trails and quick-results; and focus on the task at hand or the product of interest.

The proposed influence of Hofstede's cultural dimensions on usability has important implications. The subjective cultural approach to culturalisation, as proposed by Smith and Chang [20], and shared by others (for example [21]), is based on the belief that the cultural profile of the interface should be matched to the cultural profile of the users in order to enhance usability and performance. However, if a particular cultural profile is found to increase the usability of interfaces for all users, this would invalidate this belief. Conversely, this would still provide evidence that Hofstede's cultural dimensions are related to usability, just not in the way that was originally hypothesized.

From the above, our research objectives and hypotheses can be drawn and tested.

\subsection{Research Objectives and Hypothesis}

The aim of this research is to determine whether or not one side of each cultural dimension's dichotomy increases the general usability of user interfaces. In order to achieve this aim, the following hypotheses were tested:

H1 User interfaces designed for high power distance will be more generally usable than interfaces designed for low power distance.

H2 User interfaces designed for high uncertainty avoidance will be more generally usable than interfaces designed for low uncertainty avoidance.

H3 User interfaces designed for masculinity will be more generally usable than interfaces designed for femininity.

H4 User interfaces designed for short-term orientation will be more generally usable than interfaces designed for long-term orientation.

H5 User interfaces designed for collectivism will be as usable as interfaces designed for individualism.

\subsection{Research Design and Methodology}

Secondary data analysis was used to reanalyse data generated from a previous experiment that we conducted ${ }^{1}$. The aim of the experiment was to establish empirical evidence of a causal relationship between subjective culture and usability. As usability is tested in terms of the resultant performance achieved from using a computer to complete tasks [31], the aim of the experiment was expected to be

\footnotetext{
${ }^{1}$ The experiment is reported on in detail in a separate article [30].
} 
achieved by testing the effects of Hofstede's cultural dimensions on the performance of users using computer-based interfaces.

The experiment was conducted in the form of a formal usability test, supported by the use of questionnaires. Test subjects were sourced from a multi-cultural group of students enrolled for a third-level course in Information Systems and Technology at the University of Kwa-Zulu Natal (South Africa). Using an adapted version of Hofstede's [1] Value Survey Model, the cultural profile of each test subject was assessed. Ten test interfaces were identified using Marcus's [26] design guidelines, one for each side of each cultural dimension. Data on performance measures were collected quantitatively, using a test task instrument that comprised of test tasks and a satisfaction questionnaire. For each test task, the accuracy, speed and satisfaction of users using an interface that corresponded to their side of the cultural dimension were collected and compared to the same performance measures when using an interface with an opposing side of the cultural dimension.

Four statistical tests were used to analyse the data:

1. Related samples t-tests were used to measure whether or not there were significant differences in accuracy, speed and satisfaction levels between users using an interface that displayed design characteristics that corresponded to the subjects' cultural dimension side, compared to the same users using an interface that displayed design characteristics of an opposing cultural dimension side.

2. Independent samples t-tests on the users were used to determine whether one set of users was generally a 'better' set of users than the other. This was done by determining the average score of users of one side of the dimension using both sites, and comparing it to the average score of users of the other side of the dimension. If a significant difference was found, then it was concluded that there were variables in the test subject groups, other than culture, that were causing increased usability.

3. Independent samples t-tests on the interfaces were used to determine whether one of the sites was generally a 'better' site than the other. This was done by determining the average score achieved by all users using the first site and comparing it to the average score achieved by all users on the second site. If a significant difference was found, then it was concluded that one of the sites was better than the other, and therefore the increase in usability could be attributed to variables on the sites other than that cultural dimension.

4. Paired samples t-tests on overall usability were used to confirm the findings of the independent samples t-tests done on the users and the sites, an additional paired samples t-test was performed on the data. The data was arranged to compare the differences in scores between (1) all users using an interface with the same dimension, and (2) all users using an interface with the opposing dimension. Where the sample size of users with one side of the dimension was greater than the sample size of users with the opposing dimension, a random sample of the higher number was taken, equivalent to the smaller number in the opposing side. Because the same number of users was using the potentially superior site and the potentially inferior site, the usability difference between the sites should be nullified. Therefore, if a significant difference was not found, then the test was seen to support the findings of the independent sample t-tests described above. 


\subsection{Experimental Results}

The performance of more than 50 test subjects for four of the five cultural dimensions were measured and compared. Due to the very small sample size of short-term oriented subjects found, it was not possible to analyse the results for the time orientation dimension. The results of the four statistical tests performed on the data obtained on the measures for each cultural dimension are presented next:

1. Impact of power distance on performance: The paired samples t-test showed no significant differences in the accuracy or satisfaction levels achieved. Significant differences in speed occurred within the low power distant user group, but not within the high power distant user group. It was noted that the difference within the low power distant user group was positive, indicating that low power distant users using the low power distant site took longer to complete the tasks than when using the high power distant site. The independent samples t-test (site) indicated that irrespective of the user's side of the cultural dimension, it took longer to complete the tasks overall using the low power distant site than when using the high power distant site. This was confirmed by the lack of significant results found in the paired samples t-test used to control for usability. No significant difference was found between the two user groups.

2. Impact of uncertainty avoidance on performance: The only insignificant difference found at the $95 \%$ level in the paired samples t-test was in the accuracy scores between low uncertainty avoidant users using a low uncertainty avoidant site compared to the same users using a high uncertainty avoidant site. However, this difference fell just short of being significant in terms of the t-crit value, and could be accepted at the $94 \%$ level. It was noted that the differences found for the high uncertainty avoidant user group were exactly opposite to the differences found for the low uncertainty avoidant user group. This strongly suggested that the high uncertainty avoidant site was substantially superior to the low uncertainty avoidant site in terms of accuracy, speed and satisfaction levels. The independent samples t-tests (site) confirmed that, irrespective of the user's side of the cultural dimension, that (1) higher levels of accuracy were achieved, (2) less time was taken to complete the tasks, and (3) greater satisfaction levels were reported, when using the high uncertainty avoidant site than when using the low uncertainty avoidant site. This was confirmed by the lack of significant results found in the paired samples t-test used to control for usability. No significant difference was found between the two user groups.

3. Impact of masculinity vs. femininity on performance: The only insignificant difference found at the $95 \%$ level in the paired samples t-test was in the accuracy scores between masculine users using a masculine site compared to the same users using a feminine site. It was noted that the significant results found for the masculine user group were exactly opposite to the differences found for the feminine user group. This strongly suggested that the masculine site was substantially superior to the feminine site in terms of accuracy, speed and satisfaction levels. The independent samples t-test (site) confirmed that, irrespective of the user's side of the cultural dimension, that (1) higher levels of accuracy were achieved, (2) less time was taken to complete the tasks, and (3) greater satisfaction levels were reported, when using the masculine site than when 
using the feminine site. This was confirmed by the lack of significant results found in the paired samples t-test used to control for usability. No significant difference was found between the two user groups.

4. Impact of individualism vs. collectivism on performance: The paired samples t-test showed no significant differences in the accuracy or speed levels achieved. Significant differences in user satisfaction occurred within the individualist user group, but not within the collectivist user group. It was noted that the difference within the individualist user group was negative, indicating that individualist users using the collectivist site reported higher satisfaction levels than when using the individualist site. The independent samples t-test (site) indicated that irrespective of the user's side of the cultural dimension, greater satisfaction levels were achieved overall when using the collectivist site than when using the individualist site. This was confirmed by the lack of significant results found in the paired samples t-test used to control for usability. No significant difference was found between the two user groups.

\subsection{Analysis and Interpretation}

For every significant result obtained from the paired samples t-tests, a significant result was obtained from the independent samples t-tests for site usability differences. This indicates that the increase in performance could be attributable to the cultural dimension of the site, rather than as a result of a user using an interface with a corresponding side of a cultural dimension. In particular, the interfaces that were evaluated to be high power distant, high uncertainty avoidant, masculine or collectivist were found to be the better sites. These results support hypotheses H1, H2 and H3. In contrast to H5 (that neither collectivism nor individualism would increase the usability of the interface), the results show that collectivist sites are more generally usable that individualist sites.

These results are somewhat supported by one other study identified in the literature. Smith and Chang [20] reported that Chinese users preferred interfaces that displayed high power distant, high uncertainty avoidant, masculine and individualist characteristics. Other than the individualism/collectivism dimension, the preferred dimensions correlate to the findings of the experiment. In addition, Smith and Chang expressed surprise at the Chinese users' preference for sites that displayed individualism, in contrast to traditional perceptions of the Chinese as being a collectivist society.

Consequently, at a superficial level, the results of the experiment could allow us to accept hypotheses $\mathrm{H} 1-\mathrm{H} 3$, and reject hypothesis $\mathrm{H} 5$. $\mathrm{H} 4$ could not be tested due to the limited number of test subjects that were identified as short-term oriented.

However, it must be noted that the increase in general usability of the masculine, high power distant, collectivist and high uncertainty interfaces could also have been due to variables on the interfaces other than the cultural profile of the interfaces. For example, the user interfaces could have been more generally usable if the design of the interfaces incorporated relevant usability principles, heuristics and guidelines. Furthermore, subjective cultural dimensions other than those tested could have influenced the results of the experiment.

Consequently, to avoid a Type I error, the hypotheses cannot be accepted until further research is conducted. 


\section{Conclusions and Further Research}

Some of the arguments in the literature propose that objective, rather than subjective culture, should be accommodated into the design of user interfaces. Others argue that subjective culture is just as important as objective culture, and that the subjective cultural profile of the interface should match the subjective cultural profile of the intended users. In addition, the use of cultural dimension models as a way of managing the subjective aspects of user interface design, such as the one proposed by Hofstede [1], has been severely criticized as being stereotypical and rigid.

In contrast to the above arguments, we proposed that the interface design characteristics required to design interfaces that accommodate high power distance, high uncertainty avoidance, masculinity and short-term orientation would provide a more usable interface to all users than one that is designed to accommodate the opposing sides of these dimensions. The assumed increase in general usability was translated into the hypotheses on which this research project was based.

Secondary data analysis of an experiment previously conducted to determine the effects of Hofstede's cultural dimensions on usability has indicated that user interfaces designed to accommodate high power distance, masculinity, high uncertainty avoidance and collectivism provide better performance, irrespective of the cultural profile of the users. These results are somewhat supported by one other study [20] identified in the literature.

Although two independent studies have brought to light similar results that support our hypotheses, we noted that the differences in performance measures could have been attributable to variables other than the cultural dimensions tested that were not controlled for. This leads us to conclude that the influence of Hofstede's cultural dimensions on general usability should be considered as a topic for further research. Preliminary work on these issues is reported in [32].

\section{References}

1. Hofstede, G.: Culture's consequences (2nd ed.). Sage Publications (2001).

2. Hoft, N.: Developing a Cultural Model. In: Del Galdo, E., Nielson, J. (eds.): International User Interfaces. John Wiley and Sons, New York (1996).

3. Ciborowski, T.J.: Cross-Cultural aspects of Cognitive Functioning: Culture and Knowledge. In: Marsella, A.J., Tharp, R.G., Ciborowski, T.J. (eds): Perspectives on CrossCultural Psychology. Academic Press Inc., New York (1979).

4. Bodker, K., Pederson, J.: Workplace cultures: Looking at artifacts, symbols, and practices. In: Greenbaum, J., Kyng. M. (eds): Design at work: Cooperative Design of Computer Systems. Lawrence Erlbaum, Hillsdale, NJ (1991).

5. Borgman, C.L.: The User's Mental Model of an Information Retrieval System: an Experiment on a Prototype Online Catalog. International Journal of Man-Machine Studies, 24 (1986) 47-64.

6. Hall, E.: The Silent Language. Doubleday (1959).

7. Del Galdo, E., Nielson, J.: International User Interfaces. John Wiley and Sons (1996).

8. Victor, D.: International Business Communications. Harper Collins (1992). 
9. Trompenaars, F.: Riding the Waves of Culture. Nicholas Brealey Publishing, London (1993).

10. Evers, V.: Cultural Aspects of User Interface Understanding: An Empirical Evaluation of an E-Learning Website by International User Groups. University of Amsterdam (2001).

11. Smith A., Dunckley, L., French, T., Minocha, S., Chang, Y.: A Process Model for Developing Usable Cross-Cultural Websites. Interacting with Computers, 16 (2004) 6391.

12. ITIM. Geert Hofstede Cultural Dimensions. http://www.geert-hofstede.com/geert_hofstede_resources.shtml (retrieved January 12, 2005), (2003).

13. Marcus, A., Gould, E.W.: Crosscurrents: cultural dimensions and global web user-interface design. Interactions, 7(4) (2000) 32-46.

14. Massey, A.P., Hung, Y.C., Montoya-Weiss, M., Ramesh, V.: When culture and style aren't about clothes: perceptions of task-technology 'fit' in global virtual teams. In: Proceedings of the 2001 International ACM SIGGROUP Conference on Supporting Group Work. ACM Press, New York (2001) $207-213$.

15. Dix A., Finlay, J., Abowd, G., Beale, R.: Human-Computer Interaction. Prentice Hall International (UK), Hemel Hampstead (1998).

16. Jagne, J., Smith, S.G., Duncker, E., Curzon, P.: Cross-cultural Interface Design Strategy. Technical Report: IDC-TR-2004-006, Interaction Design Centre, Middlesex University (2004).

17. Barber, W., Badre, A.: Culturability, the merging of culture and usability. In: Proceedings of the 4th conference on Human Factors and the Web. Basking Ridge, NJ, USA (1998).

18. Chau, P.Y.K., Cole, M., Massey, A.P., Montoya-Weiss, M., O'Keefe, R.M.: Cultural differences in the online behavior of consumers. Communications of the ACM, 45(10) (2002) 138-143.

19. Marcus, A.: Cross-cultural user-interface design for work, home, and on the way. Tutorial 5, 10th Annual UPA Conference 25 - 29 June, Lake Las Vegas (2001).

20. Smith, A., Chang, Y.: Quantifying Hofstede and developing cultural fingerprints for website acceptability. In: Evers, V., Röse, K., Honold, P., Coronado, J., Day, D.L. (eds.): Proceedings of the IWIPS 2003 Conference. University of Kaiserslautern, Berlin, Germany (2003).

21. Hall, P., Lawson, C., Minocha, S.: Design patterns as a guide to the cultural localisation of software. In: In: Evers, V., Röse, K., Honold, P., Coronado, J., Day, D.L. (eds.): Proceedings of the IWIPS 2003 Conference. University of Kaiserslautern, Berlin, Germany (2003).

22. Russo, P., Boor, S.: How Fluent is your interface? Designing for international users. In: Proceedings of the INTERCHI '93 Conference on Human Factors in Computing Systems: INTERACT '93 and CHI'93. ACM Press, New York (1993) 342-347.

23. Bourges Waldegg, P., Scrivener, S.A.R.: Meaning, the central issue in cross cultural HCI design. Interacting with Computers, 9(3) (1998) 287-309.

24. Dunckley, L., Smith, A.: Cultural dichotomies in user evaluation of international software. In: Day, D., Del Galdo, E., Prahbu, G. (eds.): Proceedings of IWIPS'00, Designing for Global Markets 2. Backhouse Press Baltimore, MD (2000).

25. Carey, J.M.: Creating global software: A conspectus and review. Interacting with Computers, 9 (1998) 449-465.

26. Marcus, A.: Mapping user-interface design to cultural dimensions. Unpublished paper based on a paper prepared for a CHI 2002 Workshop and a paper prepared for Advanced Visual Interfaces, 2002. 
27. Gould, E.W., Zakaria, N., Yusof, S.A.M.: Applying culture to website design: a comparison of Malaysian and US websites. In: Proceedings of the IEEE Professional Communication Society's International Professional Communication Conference and Proceedings of the 18th Annual ACM International Conference on Computer Documentation: Technology and Teamwork. IEEE Educational Activities Department, Piscataway, NJ, USA (2000) 161-171.

28. Forer, D., Ford, G.: User performance and user interface design: Usability heuristics versus cultural dimensions. In: Mende J., Sanders, I. (eds.): Proceedings of the South African Computer Lecturer's Association 2003 Conference. Johannesburg, South Africa (2003).

29. Fitzgerald, W.: Models for Cross-Cultural Communications for Cross-Cultural Website Design. Institute for Information Technology, National Research Council Canada, (2004).

30. Ford, G., Gelderblom, J.H.: The effects of culture on performance achieved through the use of human computer interaction. In: Eloff, J., Kotzé P., Engelbrecht A., Eloff M. (eds): IT Research in Developing Countries - Proceedings of the SAICSIT 2003 Conference ACM International Conference Proceedings Series, SAICSIT, Pretoria (2003) 218-230.

31. Nielsen, J.: Usability Engineering. Academic Press (1993).

32. Ford G.: Researching the Effects of Culture on Usability. MSc Dissertation, University of South Africa (2005). 